\title{
Preparation and Characterization of Poly(divinylbenzene) Microcapsules Containing Octadecane
}

\author{
Preeyaporn Chaiyasat , Amorn Chaiyasat ${ }^{*}$, Waraporn Boontung, Supaporn Promdsorn, \\ Sutanya Thipsit \\ Department of Chemistry, Faculty of Science and Technology, Rajamangala University of Technology Thanyaburi, Thanyaburi, \\ Thailand \\ Email: ${ }^{*}$ p_chaiyasat@mail.rmutt.ac.th; ${ }^{* *}$ a_chaiyasat@mail.rmutt.ac.th
}

Received March 17 ${ }^{\text {th }}, 2011$; revised April 6 th $^{\text {th }}$ 2011; accepted April $12^{\text {th }}, 2011$

\begin{abstract}
Poly (divinylbenzene) (PDVB) microcapsules containing octadecane $(O D)(P D V B / O D)$ used as heat storage material were synthesized by suspension polymerization at $70^{\circ} \mathrm{C}$ using benzoyl peroxide and polyvinyl alcohol as initiator and stabilizer, respectively. Thermal properties and stability of PDVB/OD microcapsules were determined using differential scanning calorimeter (DSC) and thermogravimetric analyzer. The morphology and structure of microcapsules were characterized by optical microscope, scanning electron microscope and fourier transform infrared spectrophotometer. From DSC analysis, the melting temperature of encapsulated $O D\left(28^{\circ} \mathrm{C}\right)$ was almost the same as that of bulk $O D\left(30^{\circ} \mathrm{C}\right)$ while it was quite different in the case of the solidification temperature $\left(19^{\circ} \mathrm{C}\right.$ and $25^{\circ} \mathrm{C}$ for encapsulated and bulk OD, respectively). The latent heats of melting $(184.0 \mathrm{~J} / \mathrm{g}-\mathrm{OD})$ and solidification $(183.2 \mathrm{~J} / \mathrm{g}-\mathrm{OD})$ of encapsulated OD were reduced from those of bulk OD (241.7 and $247.0 \mathrm{~J} / \mathrm{g}$, respectively). However, the prepared PDVB/OD microcapsules are able to be used for heat storage applications.
\end{abstract}

Keywords: Microencapsulation, Microcapsule, Heat Storage Material, Octadecane, Suspension Polymerization, Poly (Divinylbenzene)

\section{Introduction}

The utilization of a latent heat storage system using heat storage materials or phase change materials (PCMs) is an effective method of advanced energy technologies, preserving available energy and improving its consumption. The heat storage materials are able to change the status with a convinced temperature range. They can absorb and emit the energy when the temperatures of the materials overpass the temperature of phase change during heating and cooling processes, respectively [1-10]. The thermal energy storage systems typically select the application temperatures approach phase transition temperatures of PCMs. Paraffin waxes (the linear chain hydrocarbons or n-alkanes) such as tetradecane (TD), hexadecane (HD), octadecane (OD), nonadecane (ND), and eicosane are useful as one group of numerous numbers of heat storage materials that melt and solidify at a wide range of temperatures, making them attractive for many applications [11]. These PCMs are nontoxic, non-corrosi- ve, chemically inert, easily obtained, and have no unpleasant odor. They have different phase change temperatures, melting $\left(T_{m}\right)$ and solidification $\left(T_{s}\right)$, depending on the number of carbons in their structures [9,12]. They have moderate thermal energy storage but low thermal conductivity. Therefore, they require large surface areas in applications. Encapsulation of these materials is associated with many advantages such as provide large heat transfer area, control the volume change of the storage materials as phase change occurs and protect the PCM against the influences of the outside environment $[2,6,10$, 13]. Therefore, it is more practical than the traditional PCMs in many applications such as thermal insulation [7], heat transfer [14], building materials [15] and the wall energy storage capacity [16]. A numerous number of studies have been carried out on micro and nanoencapsulation of PCMs using different polymers as the capsule shell materials. The in situ polymerization to fabricate the microcapsules and nanocapsules containing 
OD core with melamine-formaldehyde shell [17], resorcinol-modified melamine-formaldehyde shell [18] were reported. The encapsulation of n-TD core with urea-formaldehyde polymer shell were also carried out by in situ polymerization [19]. Three types of paraffin waxes (n-HD, $\mathrm{n}$-OD and n-ND) were encapsulated through complex coacervation of natural and biodegradable polymers, gum arabic-gelatin mixture [20]. Paraffin waxes encapsulated with styrene and methyl methacrylate copolymer were prepared by suspension polymerization [21]. The preparation of polystyrene (PS) microcapsules containing n-HD using casein instead of commercial stabilizer to emulsify the mixture of core materials, monomers and initiators during the suspension polymerization were also reported [22]. For textile applications, melamine formaldehyde microcapsules containing eicosane were prepared by in situ polymerization $[23,24]$ and PS microcapsules containing paraffin wax were synthesized by suspension like polymerization [25]. Polymethylmethacrylate microcapsules containing n-octaco-sane [26] and heptadecane [27] were prepared by emulsion polymerization. The capsules of PS as the shell and n-OD as the core were prepared by miniemulsion polymerization [28]. Microencapsulated n-OD with polyurea shells having different soft segments was synthesized with interfacial polycondensation [29]. To improve the shell strength, crosslinked polydivinylbenzene (PDVB) microcapsule particles with encapsulated n-HD prepared by micro-suspension polymerization utilizing the Shirasu Porous Glass membrane emulsification technique for the preparation of comparatively monodisperse DVB/HD droplets were prepared [30-32]. OD is an attractive PCM giving good capability of heat absorption and emission in appropriate phase change temperature range $\left(23-28^{\circ} \mathrm{C}\right)$ comfortable for the human body. Moreover, its latent heat $(241.2 \mathrm{~J} / \mathrm{g})$ is higher than that of the other PCMS with similar phase change temperature range [33]. As seen from the litera- tures above, a numerous of different polymers were used to prepare microencapsulated PCMs but there is no any report study on microencapsulation of OD with PDVB (the high mechanical properties polymer) shell (PDVB/OD capsules). Therefore, in this work, OD and PDVB were chosen to use as heat storage material and particle shell, respectively, of the microcapsules prepared by suspension polymerization. The morphology, chemical structure, thermal stability and thermal properties of PDVB/OD capsules were characterized by scanning electron microscope (SEM), optical microscope (OM), fourier transform infrared spectrophotometer (FT-IR), thermogravimetric analyzer (TGA) and differential scanning calorimeter (DSC), respectively.

\section{Experimental}

\subsection{Materials}

DVB (Aldrich; purity, 80\%) was washed with $1 \mathrm{~N}$ sodium hydroxide $(\mathrm{NaOH})$ and distilled water to remove polymerization inhibitors before use. Poly (vinyl alcohol) (PVA) (Aldrich; degree of saponification, 87 - 90\%) was used as received. Reagent-grade benzoyl peroxide (BPO) was purified by recrystallization. OD (Merck; 99.5\%) was used as received.

\subsection{Microcapsules Preparation}

The microcapsules of PDVB/OD were prepared by suspension polymerization as the procedure shown in Figure 1. The homogeneous organic phase of DVB and OD at the ratio of $50: 50 \% \mathrm{wt} / \mathrm{wt}$ (approximately $10 \% \mathrm{wt}$ of aqueous solution) were mixed with BPO (8\% wt of monomer) and then added to the aqueous phase containing PVA (1.5 g of PVA in $150 \mathrm{~g}$ of water). Emulsification was carried out by high shear rate at the speed of 5,000 rpm for 5 min resulting in the organic phase drop lets dispersed in the aqueous phase. The resulting emulsions were transferred to the reactor and polymerized at

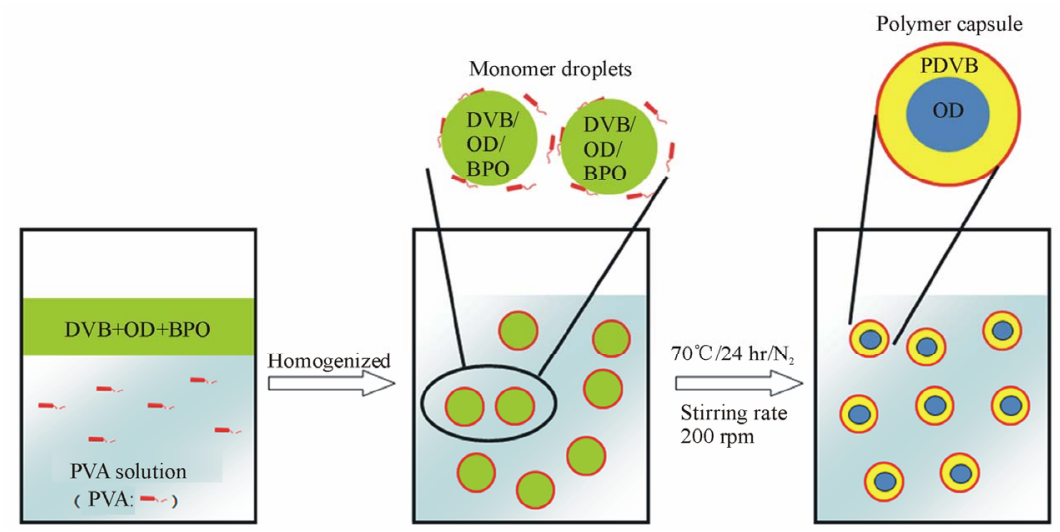

Figure 1. Schematic of the preparation process of PDVB/OD capsules with a suspension polymerization. 
$70^{\circ} \mathrm{C}$ for $24 \mathrm{~h}$ under $\mathrm{N}_{2}$ atmosphere.

\subsection{Characterization of Microcapsules}

The particle diameters of microcapsules were obtained from OM (SK-100EB \& SK-100ET, Seek, Seek Inter Corporation Ltd., Thailand) photos calculated from 200 particles by VIS Plus software. An OM and SEM (JSM-6510, JEOL, JEOL Ltd., Japan) were used to investigate the morphology of the surface and the inner structure of the microcapsules. For SEM observation, one drop of the microcapsule dispersion was placed on a nickel SEM stub and dried before Au-coated. Thermal stability of OD, PDVB and microcapsules was studied with TGA (TGA/SDTA 851 ${ }^{\mathrm{e}}$, Mettler-Toledo, Mettler-Toledo Ltd., Switzerland) using heating rate of $20^{\circ} \mathrm{C} / \mathrm{min}$. The chemical structure of the polymer was determined by FT-IR spectroscopy (PerkinElmer System 2000, PerkinElmer, USA). The dispersion sample was filtered and then kept in vacuum oven overnight. The dried sample was ground with dried potassium bromide $(\mathrm{KBr})$ powder and compressed into a disc. The pure OD was directly mixed with $\mathrm{KBr}$ before measurement.

\subsection{Thermal Properties Measurement}

The latent heats of solidification $\left(H_{s}\right)$, melting $\left(H_{m}\right), T_{s}$, and $T_{m}$ of OD encapsulated in microcapsule particles in aqueous solution (solid content: ca 10\%) were measured in an aluminum pan with a DSC (DSC 822, Metler-Toledo, Mettler-Toledo Ltd., Switzerland) under a $\mathrm{N}_{2}$ flow with the scanning temperature range and rate of $0-40^{\circ} \mathrm{C}$ and $5^{\circ} \mathrm{C} / \mathrm{min}$, respectively. To compare $H_{s}$ and $H_{m}$ of the encapsulated OD having different wt $\%$ in the capsule particles and also pure OD, the $H_{s}$ and $H_{m}$ values were used in the unit of joule per $1 \mathrm{~g}$ of encapsulated OD (J/g-OD). They were calculated from the cooling/heating peak area of DSC thermogram and OD content obtained from TGA analysis using the following equation (1).

$$
\mathrm{J} / \mathrm{g}-\mathrm{OD}=(\mathrm{A} / \mathrm{B}) \times 100
$$

where

A $=H_{s}$ or $H_{m}$ of encapsulated OD in microcapsule dispersion obtained from DSC thermogram (J/g-sample) B $=\%$ OD in microcapsule dispersion obtained from TGA thermogram

\section{Results and Discussion}

\subsection{Microcapsule Formation}

The preparation process of the PDVB capsules containing OD as heat storage material by suspension polymerization was shown in Figure 1. Aqueous phase containing PVA as stabilizer was added with oil phase consisting of OD, DVB and BPO. PVA molecules strongly adsorb onto the oil-water interface and stabilize the oil droplets generated by high shear rate. The polymerization took place within the oil droplets after the temperature reached to $70^{\circ} \mathrm{C}$. OD is well miscible with DVB monomer in the early stage of polymerization resulting in homogeneous phase in monomer droplets. When the PDVB was formed, the oil phase became gradually less compatible until reach the critical chain length of PDVB, phase separation occurred. Because the interfacial tension of PDVB-water is lower than that of OD-water, the PDVB move to the interface of oil droplets to form the polymer shell. At the end of polymerization, the polymer capsules of OD core encapsulated by PDVB shell were obtained. When the dispersions were kept for a while, all of the PDVB/OD capsules floated on the top of the dispersion solution because the total density of the capsules is lower than that of water. This indicates that the PDVB/OD capsules were successfully prepared.

\subsection{Morphology of Microcapsule and Particle Size}

The PDVB/OD microcapsules were observed by OM and SEM as shown in Figure 2. The SEM photograph indicates that most of the particle shapes were spherical with smooth outer surface even the microcapsule sizes were polydisperse consistent with the result from the optical micrograph. This is general characteristic of the polymer particles obtained from conventional suspension polymerization. Moreover, the OD core was completely encapsulated with PDVB shell as shown in the optical micrograph. The average diameter of the microcapsules is approximately $28 \mu \mathrm{m}$, measured from the optical micrographs calculated from 200 particles by VIS plus 3.0 software. Because quite small microcapsules were obtained, PDVB/OD capsules therefore afford a large surface area per unit volume. This is the reason why the microcapsules containing the heat storage material are more attractive than the pure one.

\subsection{Chemical Structure Characterization of Microcapsule}

FT-IR spectra of OD, PDVB and PDVB/OD microcapsules were shown in Figure 3. In the case of pure OD (Figure 3(a)), the characteristic absorption peak at 720 $\mathrm{cm}^{-1}$ corresponds to the in-plane rocking vibration of the methylene group. The absorption peak at 1460 and 1360 $\mathrm{cm}^{-1}$ are associated with C-H stretching vibrations of methylene bridges. The alkyl C-H stretching vibrations of methyl and methylene groups are observed at 2920 and $2850 \mathrm{~cm}^{-1}$. In the case of PDVB spectrum (Figure 3(b)), the characteristic absorption peaks at $700-750 \mathrm{~cm}^{-1}$ are benzene ring deformation vibration. The absorption 

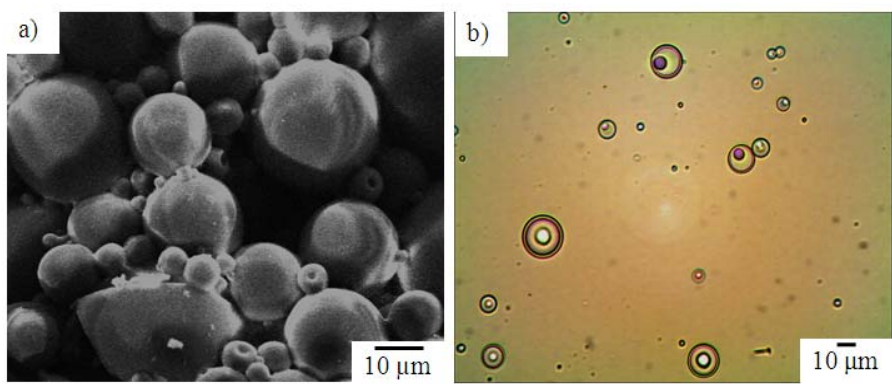

Figure 2. SEM photo (a) and optical micrograph (b) of PDVB/OD prepared by suspension polymerization.

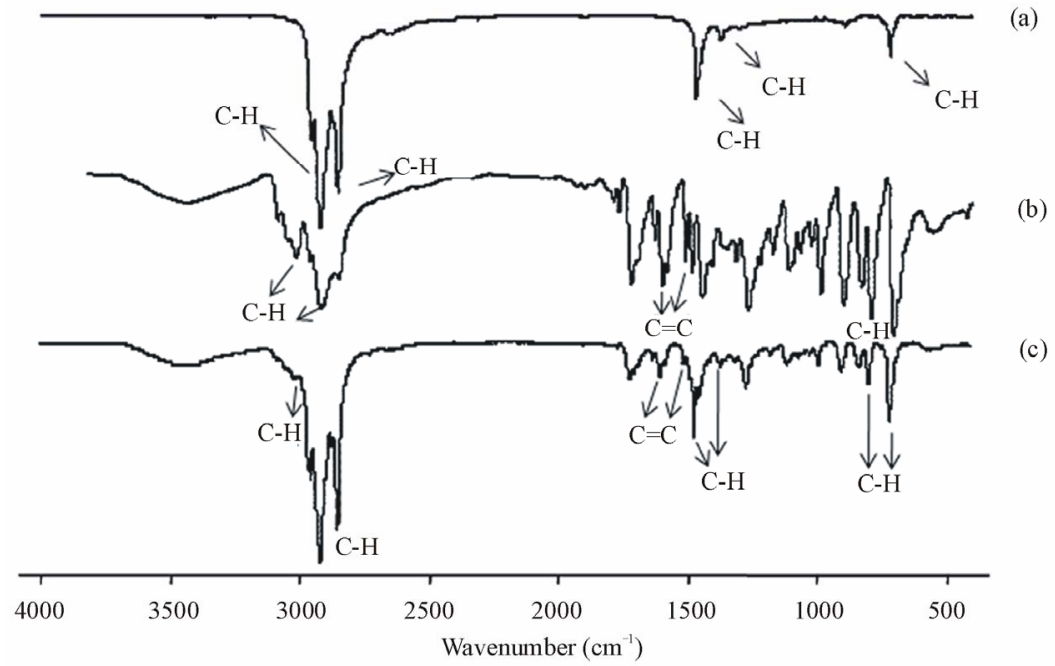

Figure 3. FT-IR spectra of OD (a), PDVB (b) and PDVB/OD capsule (c).

peaks at $1500,1600 \mathrm{~cm}^{-1}$ are associated with benzene ring $\mathrm{C}=\mathrm{C}$ stretching vibration. The aliphatic $\mathrm{C}-\mathrm{H}$ stretching vibration is observed at $2920 \mathrm{~cm}^{-1}$ whereas 3030 $\mathrm{cm}^{-1}$ showed the characteristic peaks of aromatic C-H stretching vibration. All the above characteristic peaks of bulk OD and PDVB are able to observe in the spectrum of the PDVB/OD microcapsules (Figure 3(c)), and no new peak is observed. This result indicates that the OD was incorporated in the PDVB.

\subsection{Thermal Stability of PDVB/OD Capsule}

The TGA thermograms of bulk OD, PDVB and PDVB/OD capsule were shown in Figure 4 and Table 1. The decomposition of OD started at approximately $165^{\circ} \mathrm{C}$ and completely lost its weight at $305^{\circ} \mathrm{C}$ while the decomposition temperature range of $\mathrm{PDVB}$ is $330^{\circ} \mathrm{C}$ to $495^{\circ} \mathrm{C}$. The TGA curves of OD and PDVB were sharp and showed only one step. In the case of PDVB/OD capsules TGA curve, it was mainly consisted of three steps: 50 - 124, 180 - 295 and 350 - 480 ${ }^{\circ} \mathrm{C}$ corresponding to the decomposition of water, OD and PDVB respectively. In comparison with $\mathrm{OD}$, the decomposition tem-

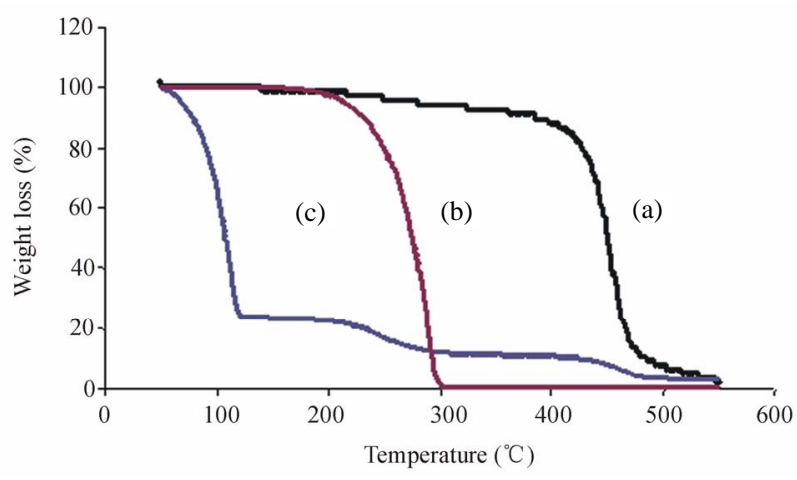

Figure 4. TGA thermograms of (a) PDVB; (b) OD and (c) PDVB/OD microcapsule.

perature of encapsulated OD was slightly higher than that of bulk OD due to the encapsulation.

\subsection{Thermal Properties of Microcapsule}

The thermal properties of the OD encapsulated in PDVB microcapsules were measured using DSC. The $H_{\mathrm{m}}(184.0$ $\mathrm{J} / \mathrm{g}-\mathrm{OD})$ and $H_{\mathrm{s}}(183.2 \mathrm{~J} / \mathrm{g}-\mathrm{OD})$ of encapsulated OD were lower than those of the bulk OD (241.0 and $247.0 \mathrm{~J} / \mathrm{g}$ of 
Table 1. TGA data of OD, PDVB and PDVB/OD microcapsule.

\begin{tabular}{ccc}
\hline & Degradation interval $\left({ }^{\circ} \mathrm{C}\right)$ & Weight loss (\%) \\
\hline OD & $162-305$ & 100 \\
PDVB & $330-495$ & 92 \\
PDVB/OD & $50-124\left(1^{\text {st }}\right.$ step $)$ & 76 \\
& $180-295\left(2^{\text {nd }}\right.$ step $)$ & 12 \\
& $350-480\left(3^{\text {rd }}\right.$ step $)$ & 10 \\
\hline
\end{tabular}

$H_{\mathrm{m}}$ and $H_{\mathrm{s}}$, respectively) as shown in Figure 5. This seems to be general case for PCMs microcapsules consistent with the other reports [17,18,22,26,27,29-32,34,35]. The possible reason of the reduction of $H_{\mathrm{m}}$ and $H_{\mathrm{s}}$ of encapsulated PCMs is that the phase separation between the polymer shell and PCM core was incomplete as in the case of PDVB/HD microcapsules prepared by suspension polymerization [32]. The capsules may have some unexhausted monomers or oligomer incorporated at the interface between polymer shell and PCM core as in the case of PS/HD microcapsules prepared by suspension polymerization [22]. They may act as the compatibilizer and help the miscibility between polymer and PCM material leading to the decreasing of the phase separation in oil phase. However, to overcome this problem, the copolymerization with more hydrophilic monomers is the good idea as copolymerization of PDVB with methyl acrylate, ethyl acrylate and butyl acrylate [32]. In the case of phase transition temperature, $T_{\mathrm{m}}$ of encapsulated OD $\left(28.3^{\circ} \mathrm{C}\right)$ was almost the same as that of bulk OD $\left(30^{\circ} \mathrm{C}\right)$. In contrast, $T_{\mathrm{s}}$ was shifted to the lower temperature comparing to bulk OD. This occurrence is namely supercooling. Supercooling leads to the reduction of the $T_{s}$ resulting in the releasing of latent heat at a lower temperature or a wider temperature range. This effect may limits to the applications. To prevent supercooling, the nucleating agents were incorporated in the PCMs core. However, increasing nucleating agent content decreased the latent heat $[36,37]$. At the present, there is still no good solution to overcome this problem. As the total surface area of the microcapsules increased comparing to bulk OD, these PDVB/OD capsules are quite acceptable for energy storage applications even having a slightly lower latent heats.

\section{Conclusions}

PDVB/OD microcapsules were successfully prepared by suspension polymerization. The optical micrographs showed that OD had been completely encapsulated inside the PDVB microcapsules with average size of $28 \mu \mathrm{m}$. The regular spherical particles with smooth outer surface were observed by SEM. All the characteristic peaks of OD and PDVB were observed in the FT-IR spectrum of

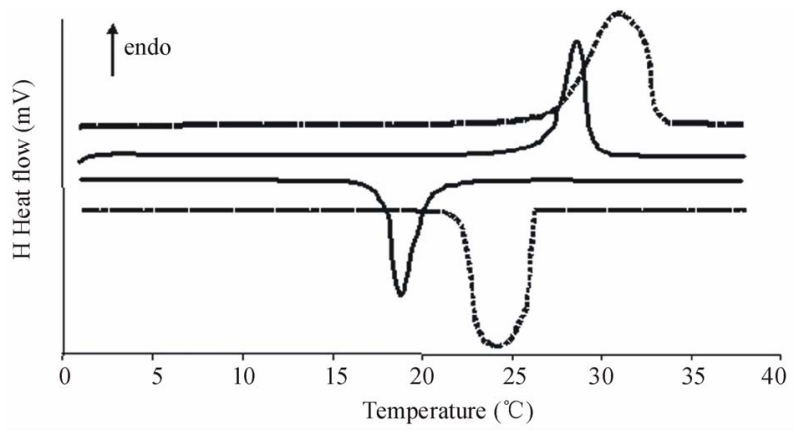

Figure 5. DSC thermograms of encapsulated OD in PDVB/OD capsule (solid line) and bulk OD (dotted line) (scanning rate $5^{\circ} \mathrm{C} / \mathrm{min}$ ).

the PDVB/OD capsules without any new peaks. The latent heats, $H_{\mathrm{m}}$ and $H_{\mathrm{s}}$, of OD in the capsule were slightly lower than those in bulk OD. As a result, the prepared PDVB/OD microcapsules are able to be used for heat storage applications because of their large surface area and still remain good thermal properties of encapsulated OD.

\section{Acknowledgements}

This work was supported by The National Research Council, Thailand (No. 23666).

\section{REFERENCES}

[1] M. Kenisarin and K. Mahkamov, "Solar Energy Storage Using Phase Change Materials," Renewable and Sustainable Energy Reviews, Vol. 11, No. 9, 2007, pp. 19131965. doi:10.1016/j.rser.2006.05.005

[2] P. Zhang, Z. W. Ma and R. Z. Wang, "An Overview of Phase Change Material Slurries: MPCS and CHS,” Renewable and Sustainable Energy Reviews, Vol. 14, No. 2, February 2010, pp. 598-614.

doi:10.1016/j.rser.2009.08.015

[3] F. Agyenim, N. Hewitt, P. Eames and M. Smyth, "A Review of Materials, Heat Transfer and Phase Change Problem Formulation for Latent Heat Thermal Energy Storage Systems (LHTESS)," Renewable and Sustainable Energy Reviews, Vol.14, No. 2, February 2010, pp. 615628.

[4] N. Zhu, Z. Ma and S. Wang, "Dynamic Characteristics and Energy Performance of Buildings Using Phase Change Materials: A Review,” Energy Convers Manage, Vol. 50, No. 12, September 2009, pp. 3169-3181. doi:10.1016/j.enconman.2009.08.019

[5] A. Sharma, V. V. Tyagi, C. R. Chen and D. Buddhi, "Review on Thermal Energy Storage with Phase Change Materials and Applications," Renewable and Sustainable Energy Reviews, Vol. 13, No. 2, February 2009, pp. 318-345. doi:10.1016/j.rser.2007.10.005

[6] S. Jegadheeswaran and S. D. Pohekar, "Performance Enhancement in Latent Heat Thermal Storage System: A Review," Renewable and Sustainable Energy Reviews, 
Vol. 13, No. 9, June 2009, pp. 2225-2244. doi:10.1016/j.rser.2009.06.024

[7] S. Mondal, "Phase Change Materials for Smart Textiles-An Overview," Applied Thermal Engineering, Vol. 28, No. 11-12, August 2008, pp. 1536-1550. doi:10.1016/j.applthermaleng.2007.08.009

[8] V. T. Tyagi and D. Buddhi, "PCM Thermal Storage in Buildings: A State of Art," Renewable and Sustainable Energy Reviews, Vol. 11, No. 6, August 2007, pp. 11461166. doi:10.1016/j.rser.2005.10.002

[9] A. M. Khudhair and M. M. Farid, “A Review on Energy Conservation in Building Applications with Thermal Storage by Latent Heat Using Phase Change Materials,” Energy Conversion Management, Vol. 45, No. 2, May 2004, pp. 263-275. doi:10.1016/S0196-8904(03)00131-6

[10] G. Sun and Z. Zhang, "Mechanical Strength of Microcapsules Made of Different Wall Materials," International Journal of Pharmaceutics, Vol. 242, January 2002, pp. 307-311. doi:10.1016/S0378-5173(02)00193-X

[11] M. M. Farid, A. M. Khudhair, S. A. K. Razack and S. Al-Hallaj, "A Review on Phase Change Energy Storage: Materials and Applications," Energy Conversion Management, Vol. 45, No. 9-10, September 2004, pp. 15971615. doi:10.1016/j.enconman.2003.09.015

[12] N. Sarier and E. Onder, "Thermal Characteristics of Polyurethane Foams Incorporated with Phase Change Materials,” Thermochimica Acta, Vol. 454, No. 2, March 2007, pp. 90-98. doi:10.1016/j.tca.2006.12.024

[13] M. N. A. Hawlader, M. S. Uddin and M. M. Khin, "Microencapsulated PCM Thermal-Energy Storage System," Applied Energy, Vol. 74, No. 1-2, January 2003, pp. 195-202. doi:10.1016/S0306-2619(02)00146-0

[14] X. Wang, J. Niu, Y. Zhang, X. Wang, B. Chen and R. Zeng, "Heat Transfer of Microencapsulated PCM Slurry Flow in a Circular Tube,” AIChE journal, Vol. 54, No. 4, 2008, pp. 1110-1120. doi:10.1002/aic.11431

[15] W. L. Wang, X. X. Yang, Y. T. Fang, J. Ding and J. Y. Yan, "Preparation and Thermal Properties of Polyethylene Glycol/Expanded Graphite Blends for Energy Storage,” Applied Energy, Vol. 86, No. 9, September 2009, pp. 1479-1483. doi:10.1016/j.apenergy.2008.12.004

[16] A. M. Borreguero, M. Carmona, M. L. Sánchez, J. L. Valverde and J. F. Rodríguez, "Improvement of the Thermal Behaviour of Gypsum Blocks by the Incorporation of Microcapsules Containing Pcms Obtained by Suspension Polimeryzation with an Optimal Core/Coating Mass Ratio,” Applied Thermal Engineering, January 2010. doi:10.1016/j.applthermaleng.2010.1001.1032

[17] X. X. Zhang, Y. F. Fan, X. M. Tao and K. L. Yick, "Fabrication and Properties of Microcapsules and Nanocapsules Containing N-octadecane,” Materials Chemistry and Physics, Vol. 88, June 2004, pp. 300-307. doi:10.1016/j.matchemphys.2004.06.043

[18] H. Z. Zhang and X. D. Wang, "Fabrication and Performances of Microencapsulated Phase Change Materials Based on N-Octadecane Core and Resorcinol-Modified Melamine-Formaldehyde Shell," Colloids and Surfaces A:
Physicochemical and Engineering Aspects, Vol. 332, No. 2-3, 15 January 2009, pp. 129-138. doi:10.1016/j.colsurfa.2008.09.013

[19] G. Fang, H. Li, F. Yang, X. Liu and S. Wu, "Preparation and Characterization of Nano-encapsulated N-tetradecane as Phase Change Material for Thermal Energy Storage," Chemical Engineering Journal, Vol. 153, No. 1-3, November 2009, pp. 217-221. doi:10.1016/j.cej.2009.06.019

[20] E. Onder, N. Sarier and E. Cimena, "Encapsulation of Phase Change Materials by Complex Coacervation to Improve Thermal Performances of Woven Fabrics," Thermochimica Acta, Vol. 467, No. 1-2, January 2008, pp. 6372. doi:10.1016/j.tca.2007.11.007

[21] L. Sánchez-Silva, J. F. Rodríguez, A. Romero, A. M. Borreguero, M. Carmona and P. Sánchez, "Microencapsulation of PCMs with a Styrene-Methyl Methacrylate Copolymer Shell by Suspension-Like Polymerisation," Chemical Engineering Journal, Vol. 157, No. 1, 15 February 2010, pp. 216-222.

[22] Y. Ai, Y. Jin, J. Sun and D. Wei, "Microencapsulation of N-hexadecane as Phase Change Material by Suspension Polymerization,” E-Polymers, No. 098, September 2007, pp. 1-9.

[23] Y. Shin, D.-I. Yoo, K. Son, "Development of Thermoregulating Textile Materials with Microencapsulated Phase Change Materials (PCM). II. Preparation and Application of PCM Microcapsules,” Journal of Applied Polymer Science, Vol. 96, No. 5, 2005, pp. 2005-2010. doi:10.1002/app.21438

[24] Y. Shin, D. Yoo and K. Son, "Development of Thermoregulating Textile Materials with Microencapsulated Phase Change Materials (PCM). IV. Performance Properties and Hand of Fabrics Treated with PCM Microcapsules,” Journal of Applied Polymer Science, Vol. 97, No. 3, 2005, pp. 910-915. doi:10.1002/app.21846

[25] P. Sánchez, M. V. Sánchez-Fernandez, A. Romero, J. F. Rodríguez and L. Sánchez-Silva, "Development of Thermo-Regulating Textiles Using Paraffin Wax Microcapsules,” Thermochimica Acta, Vol. 498, No. 1-2, January 2010, pp. 16-21. doi:10.1016/j.tca.2009.09.005

[26] A. Sarı, C. Alkan, A. Karaipekli and O. Uzun, "Microencapsulated N-Octacosane as Phase Change Material for Thermal Energy Storage,” Solar Energy, Vol. 83, No. 10, October 2009, pp. 1757-1763.

[27] A. Sarı, C. Alkan and A. Karaipekli, "Preparation, Characterization and Thermal Properties of PMMA/N-Heptadecane Microcapsules as Novel Solid- Liquid MicroPCM for Thermal Energy Storage,” Applied Energy, Vol. 87, No. 5, May 2010, pp. 1529-1534.

[28] Y. T. Fang, S. Y. Kuang, X. N. Gao and Z. G. Zhang, "Preparation and Characterization of Novel Nanoencapsulated Phase Change Materials," Energy Conversion and Management, Vol. 49, No. 12, December 2008, pp. 37043707. doi:10.1016/j.enconman.2008.06.027

[29] H. Zhang and X. Wang, "Synthesis and Properties of Microencapsulated N-Octadecane with Polyurea Shells 
Containing Different Soft Segments for Heat Energy Storage and Thermal Regulation," Solar Energy Materials \& Solar Cells, Vol. 93, No. 8, August 2009, pp. 1366-1376. doi:10.1016/j.solmat.2009.02.021

[30] P. Chaiyasat, T. Suzuki, H. Minami and M. Okubo, "Thermal Properties of Hexadecane Encapsulated in Poly (Divinylbenzene) Particles," Journal of Applied Polymer Science, Vol. 112, No. 6, 2009, pp. 3257-3266. doi:10.1002/app.29648

[31] P. Chaiyasat, Y. Ogino, T. Suzuki and M. Okubo, "Influence of Water Domain Formed in Hexadecane Core Inside Cross-linked Capsule Particle on Thermal Properties for Heat Storage Application,” Colloid Polymer Science, Vol. 286, No. 6-7, 2008, pp. 753-759. doi:10.1007/s00396-007-1831-5

[32] P. Chaiyasat, Y. Ogino, T. Suzuki, H. Minami and M. Okubo, "Preparation of Divinylbenzene Copolymer Particles with Encapsulated Hexadecane for Heat Storage Application,” Colloid Polymer Science, Vol. 286, No. 2, 2008, pp. 217-223. doi:10.1007/s00396-007-1764-Z

[33] J. Su, L. Ren and L. Wang, "Synthesis of Polyurethane MicroPCMs Containing N-Octadecane by Interfacial Polycondensation: Influence of Styrene-Maleic Anhydride as a Surfactant," Colloids and Surfaces A: Physicochemical and Engineering Aspects, Vol. 299, No. 1-3, 2007, pp. 268-275. doi:10.1016/j.colsurfa.2006.11.051

[34] J. S. Cho, A. Kwon and C. G. Cho, "Microencapsulation of Octadecane as a Phase-change Materials by Interfacial Polymerization in an Emulsion System," Colloid Polymer Science, Vol. 280, No. 3, September 2002, pp. 260-266. doi:10.1007/s00396-001-0603-X

[35] X. X. Zhang, X. M. Tao, K. L. Yick and X. C. Wang, "Structure and Thermal Stability of Microencapsulated Phase-change Materials," Colloid Polymer Science, Vol. 282, No. 4, 2004, pp. 330-336. doi:10.1007/s00396-003-0925-y

[36] X. X. Zhang, Y. F. Fan, X. M. Tao and K. L. Yick, "Crystallization and Prevention of Supercooling of Microencapsulated N-alkanes,” Journal of Colloid and Interface Science, Vol. 281, No. 2, 15 January 2005, pp. 299-306.

[37] Y. F. Fan, X. X. Zhang, X. C. Wang, J. Li and Q. B. Zhu, "Super-Cooling Prevention of Microencapsulated Phase Change Material,” Thermochimica Acta, Vol. 413, No. 12, April 2004, pp. 1-6. doi:10.1016/j.tca.2003.11.006 